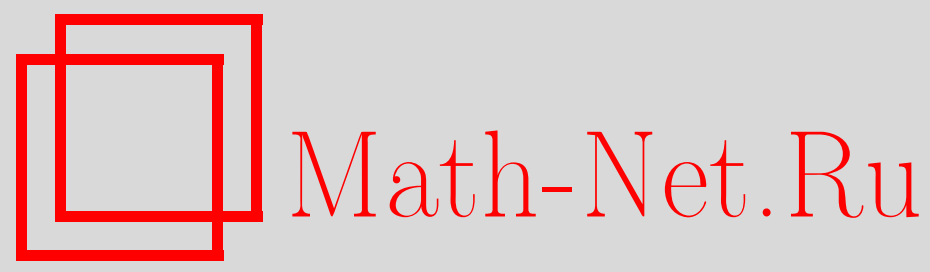

А. А. Илларионов, Оптимальное граничное управление стационарным течением вязкой неоднородной несжимаемой жидкости, Матем. заметки, 2001, том 69, выпуск 5, 666678

DOI: https://doi.org/10.4213/mzm530

Использование Общероссийского математического портала Math-Net.Ru подразумевает, что вы прочитали и согласны с пользовательским соглашением http://www.mathnet.ru/rus/agreement

Параметры загрузки:

IP : 54.210 .77 .194

26 апреля 2023 г., 02:58:00

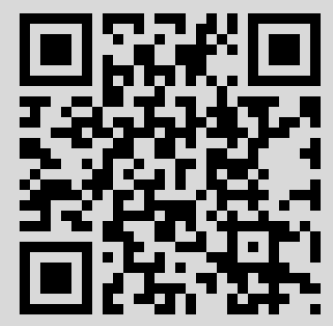




\section{ОПТИМАЛЬНОЕ ГРАНИЧНОЕ УПРАВЛЕНИЕ СТАЦИОНАРНЫМ ТЕЧЕНИЕМ ВЯЗКОЙ НЕОДНОРОДНОЙ НЕСЖИМАЕМОЙ ЖИДКОСТИ}

\section{А. А. Илларионов}

Рассматривается задача оптимального граничного управления двумерным стационарным течением вязкой неоднородной несжимаемой жидкости. Роль управления играют значения скорости на части границы рассматриваемой области. На оставшейся части границы задаются вектор скорости течения и плотность жидкости. Плотность жидкости ищется в виде некоторой скалярной функции, определяемой исходньпии данньми, от функции тока. Исследована разрешимость задачи управления и получены необходимые условия оптимальности.

Библиография: 10 названий.

\section{1. Введение}

1.1. Постановка задачи. Пусть $\Omega \subset \mathbb{R}^{2}$ ограниченная область с гранищей $\Gamma \in C^{2}$, состоящей из конечного числа связных компонент $\Gamma^{(1)}, \ldots, \Gamma^{(L)}$.

Будем рассматривать задачу оптимального управления для следующей системы уравнений, описывающей стационарное движение вязкой неоднородной несжимаемой жидкости в $\Omega$ :

$$
\begin{gathered}
-\mu \Delta u+\rho(u \nabla) u+\nabla P=\rho f, \quad \operatorname{div} u=0, \quad u \nabla \rho=0 \quad \text { в } \Omega, \\
u=y_{0}, \quad \rho=\rho_{0} \quad \text { на } \Gamma_{0}, \quad u=y_{c} \quad \text { на } \Gamma_{1}=\Gamma \backslash \Gamma_{0},
\end{gathered}
$$

где $u=\left(u_{1}, u_{2}\right)$ - вектор скорости течения, $(u \nabla) u=u_{1} \partial u / \partial x_{1}+u_{2} \partial u / \partial x_{2}, P$ - давление, $\mu>0$ - коэффициент динамической вязкости, $\rho$ - плотность жидкости, $f=\left(f_{1}, f_{2}\right)$ заданная плотность внешних сил, $y_{0}, \rho_{0}$ - заданные значения скорости и плотности на участке границы $\Gamma_{0}, y_{c}$ - значения скорости на $\Gamma_{1}$, играющие роль управления.

Предполагаем, что

$$
\begin{aligned}
& \Gamma_{0}-\text { линейно-связное замкнутое множество на } \Gamma^{(1)}, \text { meas } \Gamma_{0}>0 ; \\
& y_{0} \cdot n>0 \text { на } \Gamma_{0} \text { либо } y_{0} \cdot n<0 \text { на } \Gamma_{0} .
\end{aligned}
$$

Здесь и далее $n$ - единичный вектор внешней нормали к $\Gamma$, meas $\Gamma_{0}-$ мера Лебега множества $\Gamma_{0}$ на $\Gamma$. 
Заметим, что из условия (1.4) следует, что $\Gamma_{0}$ является участком вытекания либо втекания жидкости соответственно.

Плотность жидкости будем искать в виде: $\rho=\eta(\psi)$, где $\eta$ - скалярная функция, определяемая $y_{0}, \rho_{0}$, а $\psi$ - функция тока, т.е. $\operatorname{rot} \psi=\left(\partial \psi / \partial x_{2},-\partial \psi / \partial x_{1}\right)=u$. Такой подход был применен в [1] для доказательства разрешимости краевой задачи (1.1), (1.2). Разрешимость подобной трехмерной задачи была исследована в [2], а уравнения (1.1) с субдифференциальными граничными условиями рассмотрены в [3].

Пусть функция $\eta$ фиксирована, а граничные значения скорости на $\Gamma_{1}$ (т.е. функция $y_{c}$ ) неизвестны. Требуется отыскать течение $u$ и управление $y_{c}$ по дополнительному экстремальному условию

$$
J_{\lambda}\left(u, y_{c}\right)=J(u)+\frac{\lambda}{2} \int_{\Gamma_{1}}\left(y_{c}-y_{d}\right)^{2} d s \rightarrow \text { inf }, \quad y_{c} \in U_{a d}
$$

где $U_{a d}$ - множество допустимых управлений, $\lambda \geqslant 0, J$ - заданньй функционал, $y_{d}-$ заданная функция.

Оптимальное управление уравнениями движения вязкой однородной несжимаемой жидкости рассматривалось рядом авторов. Например, задачи с распределенным управлением в правой части для нестационарных уравнений Навье-Стокса исследованы в статьях А. В. Фурсикова [4], [5]. Подобные задачи для эволюционных и стационарных уравнений изучались в монографии Ж.-Л. Лионса [6]. Задачи граничного управления рассматривались в [7]. В этой статье доказана разрешимость поставленных задач, а с помощью принципа неопределенных множителей Лагранжа получены необходимы условия оптимальности.

Основная проблема, возникающая при исследовании задачи управления (1.1), (1.2), (1.5), обусловлена наличием дополнительного уравнения первого порядка для плотности жидкости. Последнее приводит к необходимости получения новых априорных оценок решений прямой и экстремальной задач, основанных на явном представлении плотности через функцию тока.

1.2. Определение функциональных пространств и операторов. Пусть $W^{s, p}(Q)$ - стандартное пространство Соболева, $s \in \mathbb{R}, p \geqslant 1, Q$ представляет собой либо область $\Omega$, либо гранищу $\Gamma$, либо некоторую часть границы $\Gamma$ с положительной мерой. При этом $W^{s, 2}(Q)=H^{s}(Q), W^{0, p}(Q)=L^{p}(Q)$. Через $C^{l}(\bar{\Omega})\left(C^{l}(\mathbb{R})\right)$ обозначим банахово пространство всех $l$ раз непрерывно дифференцируемых функций, которые вместе со своими производньми до $l$-го порядка включительно ограничены на $\Omega($ на $\mathbb{R})$. Аналогичные пространства вектор-функций обозначаем $\boldsymbol{H}^{s}(Q), \boldsymbol{L}^{p}(Q), \boldsymbol{C}^{l}(Q)$ и т.д. Нормы элементов в $H^{s}(Q), H^{s}(\Omega), L^{2}(Q)$ и $C^{l}(\mathbb{R})$ обозначаем через $\|\cdot\|_{s, Q},\|\cdot\|_{s}$, $\|\cdot\|_{Q}$ и $\|\cdot\|_{C^{l}}$ соответственно. Через $D^{\prime}(\Omega)$ обозначаем пространство обобщенных функций на $\Omega$.

Линейные непрерьвные операторы следа функций из $H^{1}(\Omega)$ на границу $Г$ или ее части $\Gamma_{i}$ обозначим

$$
\left.\gamma\right|_{\Gamma}: H^{1}(\Omega) \rightarrow H^{1 / 2}(\Gamma),\left.\quad \gamma\right|_{\Gamma_{i}}: H^{1}(\Omega) \rightarrow H^{1 / 2}\left(\Gamma_{i}\right), \quad i=0,1
$$


Определим гильбертовы пространства

$$
\begin{aligned}
H & =\left\{v \in \boldsymbol{H}^{1}(\Omega): \operatorname{div} v=0 \quad \text { в } \Omega, \quad \int_{\Gamma^{(i)}} v \cdot n d s=0 \quad i \in\{1, \ldots, L\}\right\}, \\
H_{0} & =\left\{v \in H:\left.\gamma\right|_{\Gamma} v=0\right\}, \quad H_{\Gamma_{0}}=\left\{v \in H:\left.\gamma\right|_{\Gamma_{0}} v=0\right\}
\end{aligned}
$$

с нормой $\boldsymbol{H}^{1}(\Omega)$ и

$$
U=\left\{g \in \boldsymbol{H}^{1 / 2}(\Gamma): \int_{\Gamma^{(i)}} g \cdot n d s=0, \quad i \in\{1, \ldots, L\}\right\}
$$

с нормой $\boldsymbol{H}^{1 / 2}(\Gamma)$. Соотношение двойственности между произвольным банаховьм либо гильбертовым пространством $X$ и его сопряженным $X^{*}$ будем обозначать через $\langle\cdot, \cdot\rangle_{X}{ }^{*} \times X$ или, если это не вызовет недоразумений, просто $\langle\cdot, \cdot\rangle$.

Введем линейньй непрерывный оператор $A: H \rightarrow H^{*}$ и трилинейные непрерывные операторы $C, C^{T}: L^{\infty}(\Omega) \times H \times H \rightarrow H^{*}$ по формулам

$$
\begin{gathered}
\langle A u, v\rangle=\sum_{i=1}^{2}\left(\nabla u_{i}, \nabla v_{i}\right)_{\Omega}, \quad\langle C(\rho, u, w), v\rangle=\int_{\Omega} \rho(u \nabla) w \cdot v d x \\
\left\langle C^{T}(\rho, u, v), w\right\rangle=\langle C(\rho, u, w), v\rangle+\langle C(\rho, w, u), v\rangle
\end{gathered}
$$

для любых $u, w, v \in H, \rho \in L^{\infty}(\Omega)$. Заметим, что существует $\gamma>0$ такая, что

$$
\gamma\|u\|_{1}^{2} \leqslant\langle A u, u\rangle \quad \forall u \in H_{0} .
$$

Через $C_{1}, C_{2}, \ldots$ будем обозначать константы, значения которых являются неизменными на протяжении доказательства одной теоремы, леммы и.т.д.

\section{2. Слабое решение краевой задачи}

2.1. Определение слабого решения. Предполагаем, что

$$
\rho_{0} \in C^{0}\left(\Gamma_{0}\right), \quad 0<\rho_{0}(x), \quad x \in \Gamma_{0}, \quad y_{0} \in \boldsymbol{H}^{1 / 2}\left(\Gamma_{0}\right) .
$$

Пусть $x_{0}-$ начало кривой $\Gamma_{0}$. Для любого $u \in H$ существует единственная скалярная функция $\psi \in H^{2}(\Omega)$ (см., например, [1]) такая, что

$$
\operatorname{rot} \psi=\left(\frac{\partial \psi}{\partial x_{2}},-\frac{\partial \psi}{\partial x_{1}}\right)=u \quad \text { в } \Omega, \quad \psi(x)=\int_{\Gamma_{0}\left(x_{0}, x\right)} u \cdot n d s, \quad x \in \Gamma_{0},
$$

где $\Gamma_{0}\left(x_{0}, x\right)$ - часть кривой $\Gamma_{0}$, соединяющая точки $x_{0}$ и $x$. Введем оператор $N: H \rightarrow$ $H^{2}(\Omega)$, которьй каждому $u \in H$ ставит в соответствие $\psi \in H^{2}(\Omega)$, удовлетворяющую уравнениям $(2.2)$. Рассмотрим функцию $\psi_{0}$, определяемую формулой

$$
\psi_{0}(x)=\int_{\Gamma_{0}\left(x_{0}, x\right)} y_{0} n d s, \quad x \in \Gamma_{0} .
$$

Так как $y_{0} \cdot n \in \boldsymbol{H}^{1 / 2}\left(\Gamma_{0}\right)$, то $\psi_{0} \in H^{3 / 2}\left(\Gamma_{0}\right) \subset C^{0}\left(\Gamma_{0}\right)$. Из условий $(1.3),(1.4)$ следует строгая монотонность $\psi_{0}$ на $\Gamma_{0}$; значит, существует $\psi_{0}^{-1} \in C^{0}(B)$, где $B=\left\{\psi_{0}(x)\right.$, $\left.x \in \Gamma_{0}\right\}$. Следовательно, при вьполнении условий (1.3), (1.4), (2.1) найдется функция $\eta$ такая, что

$$
\eta \in C^{0}(\mathbb{R}), \quad 0<\eta(\xi) \quad \forall \xi \in \mathbb{R}, \quad \eta(\xi)=\rho_{0}\left(\psi_{0}^{-1}(\xi)\right), \quad \xi \in B .
$$

Далее считаем $\eta$, удовлетворяющую (2.3), фиксированной. 
ЗАмЕчАниЕ. Далеев ряде случаев нам потребуется дополнительная гладкость функции $\eta$. Нетрудно проверить, что если в дополнение к условиям $(1.3),(1.4),(2.1)$ $\rho \in C^{k}(\mathbb{R}), \Gamma_{0} \in C^{k},\left(y_{0} \cdot n\right) \in C^{k-1}\left(\Gamma_{0}\right)(k \geqslant 1)$, то найдется $\eta \in C^{k}(\mathbb{R})$, удовлетворяющая соотношениям (2.3).

Для любого $u \in H$ имеет место равенство

$$
\int_{\Omega} u \nabla \varphi \cdot \eta(N u) d \Omega=0 \quad \forall \varphi \in H^{1}(\Omega),\left.\quad \gamma\right|_{\Gamma} \varphi=0
$$

которое непосредственно проверяется для $\eta \in C^{1}(\mathbb{R})$, а в случае $\eta \in C^{0}(\mathbb{R})$ получается регуляризацией функции $\eta$. Если дополнительно $\left.\gamma\right|_{\Gamma_{0}} u \cdot n=y_{0} \cdot n$, то из построения функции $\eta$ вытекает

$$
\eta(N u)=\rho_{0} \quad \text { на } \Gamma_{0}
$$

Положим

$$
g(x)= \begin{cases}y_{0}(x), & \text { если } x \in \Gamma_{0}, \\ y_{c}(x), & \text { если } x \in \Gamma_{1} .\end{cases}
$$

ОПРЕДЕЛЕНИЕ. Пару $u \in H, \rho=\eta(N u)$ назовем слабым решением краевой задачи (1.1), (1.2), если

$$
\begin{gathered}
\mu A u+C(\eta(N u), u, u)=\eta(N u) f \quad \text { в } H_{0}^{*},\left.\quad \gamma\right|_{\Gamma} u=g, \\
\eta \in C^{0}(\mathbb{R}), \quad \eta(N u)=\rho_{0} \quad \text { на } \Gamma_{0} .
\end{gathered}
$$

Пусть $u \in C^{2}(\bar{\Omega}), \rho \in C^{1}(\bar{\Omega})$ - решение системы $(1.1),(1.2)$. Если $u \neq 0$ в $\Omega$, $u \in C^{1}(\bar{\Omega})$, то общее решение уравнения $u \nabla \rho=0$ в $\Omega$ имеет вид

$$
\rho=\tilde{\eta}(\psi), \quad \operatorname{rot} \psi=\left(\frac{\partial \psi}{\partial x_{2}},-\frac{\partial \psi}{\partial x_{1}}\right)=u
$$

где $\tilde{\eta}$ - произвольная непрерьвно дифференцируемая функция. Тогда систему (1.1), (1.2) можно записать в виде

$$
\begin{array}{cll}
-\mu \Delta u+\tilde{\eta}(N u)(u \nabla) u+\nabla p=\tilde{\eta}(N u) f, & \operatorname{div} u=0 \quad \text { в } \Omega, \\
u=g \quad \text { на } \Gamma, \quad \tilde{\eta}(N u)=\rho_{0} & \text { на } \Gamma_{0} .
\end{array}
$$

Скалярно в $L^{2}(\Omega)$ умножая первое соотношение в $(2.8)$ на произвольную $v \in H_{0}$ и пользуясь формулой интегрирования по частям, получаем, что $u$ удовлетворяет первому соотношению в (2.6).

Обратно, пусть $u \in H, \rho=\eta(N u)$ - слабое решение (1.1), (1.2); тогда, как и в [8], заключаем, что найдется $P \in L^{2}(\Omega)$ такой, что

$$
-\mu \Delta u+\rho(u \nabla) u+\nabla P=\rho f \quad \text { в } D^{\prime}(\Omega) .
$$

Из формул $(2.4),(2.5)$ вытекает $\operatorname{div}(u \rho)=0$ в $D^{\prime}(\Omega), \rho=\rho_{0}$ на $\Gamma_{0}$. 
2.2. Существование слабого решения. Нашей ближайшей целью является доказательство разрешимости задачи (2.6) для любых $g \in U, \eta \in C^{0}(\mathbb{R})$. Подобньй результат был получен в [1] для случая $g \in U \cap C^{1}(\Gamma), \eta \in C^{0, \alpha}(\mathbb{R}), \alpha>0$.

ЛЕмма 2.1. Oператор $N$ непрерывен.

ДоказАтельСтво. Рассмотрим оператор

$$
\operatorname{rot}: H_{x_{0}}=\left\{\psi \in H^{2}(\Omega): \psi\left(x_{0}\right)=0\right\} \rightarrow H .
$$

Очевидно, что если $\operatorname{rot} \psi=0$, то $\psi=0$, т.е. $\operatorname{rot}$ является инъективньп оператором. Пусть $u \in H$; из определения оператора $N$ следует, что $\psi=N u \in H_{x_{0}}$, rot $\psi=u$, т.е. оператор rot сюръективен. Из непрерьвности вложения $H^{2}(\Omega) \subset C^{0}(\bar{\Omega})$ вытекает, что пространство $H_{x_{0}}$ гильбертово с нормой $\|\cdot\|_{2}$. Таким образом, непрерьвньй линейный оператор rot осуществляет биективное отображение $H_{x_{0}}$ на $H$; следовательно, по теореме Банаха [9] обратньй к нему оператор $N$ также является непрерывным.

Для краткости введем операторы $\widetilde{C}: H \times H \times H \rightarrow H^{*}, F: H \rightarrow H^{*}$ с помощью формул

$$
\widetilde{C}(u, w, v)=C(\eta(N u), w, v), \quad\langle F(u), v\rangle=\int_{\Omega} \eta(N u) f \cdot v d \Omega \quad \forall u, w, v \in H .
$$

Имеет место равенство

$$
\langle\widetilde{C}(u, u, v), v\rangle=0 \quad \forall v \in H_{0}, u \in H
$$

которое проверяется непосредственно для $\eta \in C^{1}(\mathbb{R})$, а для $\eta \in C^{0}(\mathbb{R})$ получается регуляризацией функции $\eta$.

Пусть $X, Y$ - банаховы пространства. Будем говорить, что оператор $\Phi: X \rightarrow Y$ компактныци, если он ограниченные множества переводит в компактные; слабо непрерывный, если он слабо сходящиеся последовательности переводит в слабо сходящиеся; усиленно непрерывны й, если он слабо сходящиеся последовательности переводит в сильно сходящиеся. Очевидно, что если пространства $X, Y$ рефлексивные, то для того чтобы оператор $\Phi: X \rightarrow Y$ был усиленно непрерьвньг, необходимо и достаточно, чтобы он был слабо непрерьвньп и компактным.

Лемма 2.2. Пусть $f \in \boldsymbol{L}^{p}(\Omega), 1<p, \eta \in C^{0}(\mathbb{R})$. Тогда операторы $\widetilde{C}, F$ усиленно непрерывные.

ДокАЗАтЕльство. Достаточно показать, что операторы $\widetilde{B}, F$ слабо непрерывные и компактные. Пусть $u_{k}, w_{k}, v_{k}, \rightarrow u, w, v$ слабо в $H$. Из компактности вложений

$$
\boldsymbol{H}^{1}(\Omega) \subset \boldsymbol{L}^{q}(\Omega), \quad H^{2}(\Omega) \subset W^{1, q}(\Omega), \quad 1 \leqslant q<\infty,
$$

непрерьвности вложения $W^{1, r}(\Omega) \subset C^{0}(\bar{\Omega})$ и леммы 2.1 вытекает, что $w_{k}, v_{k} \rightarrow w, v$ сильно в $L^{q}(\Omega), N u_{k} \rightarrow N u$ сильно в $C^{0}(\bar{\Omega}) ;$ следовательно, $\rho_{k}=\eta\left(N u_{k}\right) \rightarrow \rho=\eta(N u)$ в $C^{0}(\bar{\Omega})$. Тогда, используя неравенство Гёльдера, нетрудно показать, что

$$
\int_{\Omega} \rho_{k}\left(w_{k} \nabla v_{k}\right) \cdot y d \Omega+\int_{\Omega} \rho_{k} f \cdot y d \Omega \rightarrow \int_{\Omega} \rho(w \nabla v) \cdot y d \Omega+\int_{\Omega} \rho f \cdot y d \Omega
$$


для любого $y \in H$. Следовательно, операторы $\widetilde{B}, F$ слабо непрерывные. Используя неравенство Гёльдера и непрерьвность вложений (2.10), нетрудно проверить, что если множества $M_{1} \subset H \times H \times H, M_{2} \subset H$ ограничены, то множества

$$
\widetilde{B}\left(M_{1}\right)=\left\{\widetilde{B}(u, w, y):(u, w, y) \in M_{1}\right\}, \quad F\left(M_{2}\right)=\left\{F(u): u \in M_{2}\right\}
$$

ограничены в норме пространства $\left(L^{r}(\Omega)\right)^{*}(2<r<\infty)$. В силу компактности вложения $\left(L^{r}(\Omega)\right)^{*} \subset H^{*} M_{1}, M_{2}$ - компактные множества пространства $H^{*}$. Значит, операторы $\widetilde{B}, F$ компактные. Лемма доказана.

Выберем вектор-функцию $u_{g} \in H$ из условий

$$
\left\langle A u_{g}, v\right\rangle=0 \quad \forall v \in H_{0},\left.\quad \gamma\right|_{\Gamma} u_{g}=g .
$$

Такая $u_{g}$ сушествует и единственна [8], причем $\left\|u_{g}\right\|_{1} \leqslant C_{1}(\Omega)\|g\|_{1 / 2, \Gamma}$. Из леммы 2.1 вытекает, что функция $b=N u_{g}$ удовлетворяет оценке

$$
\|b\|_{2} \leqslant C_{2}(\Omega)\left\|u_{g}\right\|_{1} \leqslant C_{1} C_{2}\|g\|_{1 / 2, \Gamma}
$$

В $\left[8\right.$, с. 366] построено множество “срезающих" функций $\theta_{\varepsilon} \in C^{2}(\bar{\Omega}), \varepsilon>0$, таких, что функция $u_{\varepsilon}=\operatorname{rot}\left(b \cdot \theta_{\varepsilon}\right)$ удовлетворяет соотношениям

$$
\begin{gathered}
\int_{\Omega}\left|(v \nabla) u_{\varepsilon} \cdot v\right| d x \leqslant C_{3}(\Omega)\left(\sum_{i, j=1}^{2}\left\|\frac{\partial^{2} b}{\partial^{2} x_{i} x_{j}}\right\|_{L^{3 / 2}\left(\Omega_{\varepsilon}\right)}+\varepsilon\|b\|_{2}\right)\|v\|_{1}^{2} \quad \forall v \in H_{0} \\
\left.\gamma\right|_{\Gamma} u_{\varepsilon}=g \quad \text { на } \Gamma
\end{gathered}
$$

где $\Omega_{\varepsilon}=\{x \in \Omega: \operatorname{dist}(x, \Gamma) \leqslant 2 \exp (-1 / \varepsilon)\}$. Здесь $\operatorname{dist}(x, \Gamma)-\operatorname{paccтояние~от~точки~} x$ до гранищы Г. Используя $(2.11),(2.12)$ и неравенство Гёльдера, нетрудно получить оценку

$$
\begin{gathered}
\int_{\Omega}\left|(v \nabla) u_{\varepsilon} \cdot v\right| d x \leqslant \delta_{\varepsilon}(g) \cdot\|v\|_{1} \quad \forall v \in H_{0} \\
\delta_{\varepsilon}(g)=C_{4}(\Omega)\left(\varepsilon+\left(\operatorname{meas} \Omega_{\varepsilon}\right)^{1 / 6}\right)\|g\|_{1 / 2, \Gamma}
\end{gathered}
$$

Из построения функции $u_{\varepsilon}$ вытекает, что

$$
\left\|u_{\varepsilon}\right\|_{1} \leqslant C_{5}(\Omega)\|g\|_{1 / 2, \Gamma} \cdot\left\|\theta_{\varepsilon}\right\|_{C^{2}(\Omega)} .
$$

Tеорема 2.1. Пусть $f \in \boldsymbol{L}^{p}(\Omega), 1<p, \eta \in C^{0}(\mathbb{R})$. Тогда для любого $g \in U$ существует функиия $u \in H$, удовлетворяющая уравнениям (2.6), причем имеет место априорная оиенка

$$
\|u\|_{1} \leqslant\left\|u_{\varepsilon}\right\|_{1}+\frac{\mu\left\|u_{\varepsilon}\right\|_{1}+C_{1}\|\eta\|_{C^{0}} \cdot\left(\|f\|_{L^{p}(\Omega)}+\left\|u_{\varepsilon}\right\|_{1}^{2}\right)}{\gamma \mu-\delta_{\varepsilon}(g)\|\eta\|_{C^{0}}},
$$

верная для любого $u_{\varepsilon} \in H$, удовлетворяющего $(2.13)$, с $\delta_{\varepsilon}(g)<\gamma \mu /\|\eta\|_{C^{0}}, C_{1}=$ $C_{1}(\Omega, p)$. 
ДокаЗАтельство. Решение ищем в виде: $u=u_{\varepsilon}+u_{0}$, где $u_{0} \in H_{0}-$ новая неизвестная функция, $u_{\varepsilon}$ удовлетворяет условиям $(2.13)$, в которых $\delta_{\varepsilon}(g)<\gamma \mu /\|\eta\|_{C^{0}}$. Тогда соотношения (2.6) эквивалентны уравнению

$$
\begin{aligned}
& \mu A u_{0}+\widetilde{C}\left(u_{\varepsilon}+u_{0}, u_{0}+u_{\varepsilon}, u_{0}\right)+\widetilde{C}\left(u_{\varepsilon}+u_{0}, u_{0}, u_{\varepsilon}\right) \\
& =F\left(u_{\varepsilon}+u_{0}\right)-\mu A u_{\varepsilon}-\widetilde{C}\left(u_{\varepsilon}+u_{0}, u_{\varepsilon}, u_{\varepsilon}\right) \quad \text { в } H_{0}^{*} .
\end{aligned}
$$

Введем нелинейньй оператор $T: H_{0} \rightarrow H_{0}$, который каждому $w \in H_{0}$ ставит в соответствие элемент $u_{0} \in H_{0}$, являющийся решением уравнения

$$
a_{w}\left(u_{0}, v\right)=\left\langle f_{w}, v\right\rangle \quad \forall v \in H_{0}
$$

где

$$
\begin{aligned}
\left\langle f_{w}, v\right\rangle= & \left\langle F\left(u_{\varepsilon}+w\right), v\right\rangle-\mu\left\langle A u_{\varepsilon}, v\right\rangle-\left\langle\widetilde{C}\left(u_{\varepsilon}+w, u_{\varepsilon}, u_{\varepsilon}\right), v\right\rangle, \\
a_{w}\left(u_{0}, v\right)= & \mu\left\langle A u_{0}, v\right\rangle+\left\langle\widetilde{C}\left(u_{\varepsilon}+w, w+u_{\varepsilon}, u_{0}\right), v\right\rangle \\
& +\left\langle\widetilde{C}\left(u_{\varepsilon}+w, u_{0}, u_{\varepsilon}\right), v\right\rangle \quad \forall v \in H_{0} .
\end{aligned}
$$

Очевидно, что билинейная форма $a_{w}$ непрерьвна на $H_{0} \times H_{0}$; более того, из $(1.6),(2.9)$ и условия (2.13) вытекает, что

$$
0<\left(\mu \gamma-\delta_{\varepsilon}(g)\|\eta\|_{C^{0}}\right)\|v\|_{1}^{2} \leqslant a_{w}(v, v) \quad \forall v \in H_{0}, \quad v \neq 0
$$

следовательно, форма $a_{w}$ коэрцитивна на $H_{0} \times H_{0}$. Нетрудно показать, что линейньй функционал $f_{w}$ непрерывен на $H_{0}$, причем

$$
\left\langle f_{w}, v\right\rangle \leqslant \mu\left\|u_{\varepsilon}\right\|_{1} \cdot\|v\|_{1}+C_{1}\|\eta\|_{C^{0}} \cdot\|v\|_{1}\left(\|f\|_{L^{p}(\Omega)}+\left\|u_{\varepsilon}\right\|_{1}^{2}\right) \quad \forall v \in H_{0} .
$$

Тогда по теореме Лакса-Мильграмма для любого $w \in H_{0}$ существует единственный $u_{0} \in H_{0}$, удовлетворяющий уравнению (2.17), причем

$$
\left\|u_{0}\right\|_{1} \leqslant \frac{\mu\left\|u_{\varepsilon}\right\|_{1}+C_{1}\|\eta\|_{C^{0}}\left(\|f\|_{L^{p}(\Omega)}+\left\|u_{\varepsilon}\right\|_{1}^{2}\right)}{\gamma \mu-\delta_{\varepsilon}(g)\|\eta\|_{C^{0}}} .
$$

Следовательно, оператор $T$ введен корректно. Очевидно, что (2.16) эквивалентно уравнению $T\left(u_{0}\right)=u_{0}$. Для доказательства существования неподвижной точки оператора $T$, учитьвая оценку (2.18), по теореме Шаудера о существовании неподвижной точки у вполне непрерывного оператора $[9$, с. 411] достаточно показать, что оператор $T$ компактен. Пусть $w_{m} \rightarrow w_{0}$ слабо в $H_{0}, T\left(w_{m}\right)=u_{m}, T\left(w_{0}\right)=u_{0}$. Вычитая из $(2.17)$, записанного для $w=w_{m}$, то же самое уравнение, записанное для $w=w_{0}$, получаем, что

$$
\begin{aligned}
& \mu A\left(u_{m}-u_{0}\right)+\left(\widetilde{C}\left(u_{\varepsilon}+w_{m}, w_{m}+u_{\varepsilon}, u_{0}\right)-\widetilde{C}\left(u_{\varepsilon}+w_{0}, w_{0}+u_{\varepsilon}, u_{0}\right)\right) \\
& \quad+\left(\widetilde{C}\left(u_{\varepsilon}+w_{m}, u_{0}+u_{\varepsilon}, u_{\varepsilon}\right)-\widetilde{C}\left(u_{\varepsilon}+w_{0}, u_{0}+u_{\varepsilon}, u_{\varepsilon}\right)\right) \\
& \quad+\widetilde{C}\left(u_{\varepsilon}+w_{m}, w_{m}+u_{\varepsilon}, u_{m}-u_{0}\right)-\widetilde{C}\left(u_{\varepsilon}+w_{m}, u_{0}-u_{m}, u_{\varepsilon}\right) \\
& =F\left(u_{\varepsilon}+w_{m}\right)-F\left(u_{\varepsilon}+w_{0}\right) \quad \text { в } H_{0}^{*} .
\end{aligned}
$$


Скалярно умножая полученное вьше уравнение на $\left(u_{m}-u_{0}\right) \in H_{0}$ и пользуясь свойствами (1.6), (2.9), условием (2.13) и леммой 2.2, приходим к соотношению

$$
\begin{aligned}
& \left(\mu \gamma-\delta_{\varepsilon}(g)\|\eta\|_{C^{0}}\right)\left\|u_{m}-u_{0}\right\|_{1} \leqslant\left\|F\left(u_{\varepsilon}+w_{m}\right)-F\left(u_{\varepsilon}+w_{0}\right)\right\|_{H^{*}} \\
& \quad+\left\|\left(\widetilde{C}\left(u_{\varepsilon}+w_{m}, w_{m}+u_{\varepsilon}, u_{0}\right)-\widetilde{C}\left(u_{\varepsilon}+w_{0}, w_{0}+u_{\varepsilon}, u_{0}\right)\right)\right\|_{H^{*}} \\
& \quad+\left\|\left(\widetilde{C}\left(u_{\varepsilon}+w_{m}, u_{0}+u_{\varepsilon}, u_{\varepsilon}\right)-\widetilde{C}\left(u_{\varepsilon}+w_{0}, u_{0}+u_{\varepsilon}, u_{\varepsilon}\right)\right)\right\|_{H^{*}} \rightarrow 0 .
\end{aligned}
$$

Следовательно, $u_{m}=T\left(w_{m}\right) \rightarrow u_{0}=T\left(w_{0}\right)$ сильно в $H$ при $m \rightarrow \infty$. Доказали, что оператор $T$ усиленно непрерывный, а значит, он вполне непрерывный. Для получения оценки (2.15) достаточно заметить, что $\|u\|_{1} \leqslant\left\|u_{\varepsilon}\right\|_{1}+\left\|u_{0}\right\|_{1}$, и воспользоваться неравенством (2.18). Теорема доказана.

\section{3. Оптимальное граничное управление системой $(1.1),(1.2)$}

3.1. Постановка экстремальной задачи. Введем в рассмотрение множество допустимых управлений $U_{a d}$, удовлетворяющее условию

(а) $U_{a d} \subset U, g=y_{0}$ на $\Gamma_{0} \forall g \in U_{a d}, U_{a d} \neq \varnothing, U_{a d}$ - замкнутое вьпуклое множество. Определим множество допустимых пар “течение-управление" $S$ и функционал качества $J_{\lambda}: H \times H^{1 / 2}(\Gamma) \rightarrow \mathbb{R}$ по формулам

$$
\begin{gathered}
S=\left\{(u, g) \in H \times U_{a d}: \mu A u+\widetilde{B}(u, u, u)=F(u) \quad \text { в } H_{0}^{*},\left.\quad \gamma\right|_{\Gamma} u=g\right\}, \\
J_{\lambda}(u, g)=J(u)+\frac{\lambda}{2}\left\|g-y_{d}\right\|_{\Gamma_{1}}^{2} \quad \forall u \in H, \quad g \in H^{1 / 2}(\Gamma),
\end{gathered}
$$

где $\lambda \geqslant 0, y_{d} \in H^{1 / 2}\left(\Gamma_{1}\right)$ - заданньй элемент, $J: H \rightarrow \mathbb{R}$ - заданный функционал.

Обозначим через $\widetilde{S}$ множество допустимых “течений”, т.е. $\widetilde{S}$ состоит из всех функций $u \in H$ таких, что $\left(u,\left.\gamma\right|_{\widetilde{\Gamma}} u\right) \in S$. Используя лемму 2.2 и условие $(\mathrm{a})$, нетрудно показать, что множества $S$ и $\widetilde{S}$ слабо замкнуты.

Далее считаем, что

(b) $J(v) \geqslant 0 \forall v \in H, J$ слабо полунепрерывен снизу на $\widetilde{S}$.

ЗАдАчА 1 . Найти $\left(u_{*}, g_{*}\right) \in S: J_{\lambda}\left(u_{*}, g_{*}\right) \leqslant J_{\lambda}(u, g) \forall(u, g) \in S$.

Теорема 3.1. Пусть выполняются условия (a), (b), $f \in L^{p}(\Omega), 1<p, \eta \in C^{0}(\mathbb{R})$. Тогда если множество $U_{a d}$ ограничено либо функиионал $J$ коэриитивен на $\widetilde{S}$, то существует решение әкстремальной задачи 1.

ДокАЗАТЕЛЬСтво. Согласно теореме 2.1 множество $S$ непусто. Пусть

$$
J_{\lambda}\left(u_{m}, g_{m}\right) \rightarrow J^{*}=\inf _{(u, g) \in S} J_{\lambda}(u, g), \quad\left(u_{m}, g_{m}\right) \in S
$$

Если множество $U_{a d}$ ограничено, то $\left\|g_{m}\right\|_{1,2, \Gamma} \leqslant C_{1}$ и из оценок $(2.14),(2.15)$ вытекает $\left\|u_{m}\right\|_{1} \leqslant C_{2}$. Если функционал $J$ коэрцитивен на $\widetilde{S}$, то $\left\|u_{m}\right\|_{1} \leqslant C_{2}^{\prime}$; следовательно, 
$\left\|g_{m}\right\|_{1,2, \Gamma} \leqslant C_{1}^{\prime}$. Постоянные $C_{1}, C_{2}, C_{1}^{\prime}, C_{2}^{\prime}$ не зависят от $m$. Значит, можно извлечь подпоследовательности

$$
u_{m_{k}} \rightarrow u_{*} \text { слабо в } H, \quad g_{m_{k}} \rightarrow g_{*} \text { слабо в } H^{1 / 2}(\Gamma), \quad\left(u_{m_{k}}, g_{m_{k}}\right) \in S .
$$

Из слабой замкнутости множества $S$ вытекает, что $\left(u_{*}, g_{*}\right) \in S$. Следовательно,

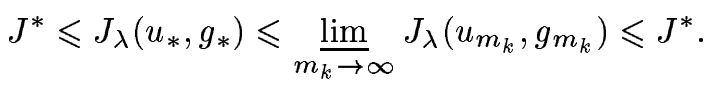

То есть $J_{\lambda}\left(u_{*}, g_{*}\right)=J^{*},\left(u_{*}, g_{*}\right)$ - решение задачи 1 . Теорема доказана.

3.2. Примеры функционалов качества. В качестве возможных $J$, с учетом физического смысла рассматриваемых задач, можно выбирать $J=J_{i}, 1 \leqslant i \leqslant 5$, где

$$
\begin{aligned}
J_{1}(u) & =\frac{1}{s} \int_{\Omega}\left|u-u_{d}\right|^{s} d \Omega, & J_{2}(u) & =\frac{1}{2} \int_{\Omega}|\operatorname{rot} u|^{2} d \Omega, \\
J_{3}(u) & =\frac{1}{s} \int_{\Omega}\left|\eta(N u)-\rho_{d}\right|^{s} d \Omega, & J_{4}(u) & =\frac{\mu}{2} \int_{\Omega}\left|(\nabla u)+(\nabla u)^{T}\right|^{2} d \Omega, \\
J_{5}(u) & =\frac{1}{s} \int_{\Omega^{\prime}}\left|\eta^{\prime}(N u) u\right|^{s} d \Omega, & u_{d}, \rho_{d} & \in L^{s}(\Omega), \quad 2 \leqslant s<\infty, \quad \Omega^{\prime} \subset \Omega .
\end{aligned}
$$

Функционалы $J_{1}, J_{2}, J_{3}$ описывают отклонение вектора скорости течения от заданного, завихренность поля скоростей и отклонение плотности жидкости от заданной соответственно. Следует выбирать $J=J_{4}$, если необходимо минимизировать диссипацию энергии в жидкости, возникающую за счет сил вязкого трения, и $J=J_{5}$, если необходимо минимизировать градиент плотности жидкости в некоторой подобласти $\Omega^{\prime} \subset \Omega$.

Очевидно, что при выполнении условия (а) функционалы $J_{i}, i=1,2,3,4$, слабо полунепрерьвны снизу на множестве $\widetilde{S}$. Слабая непрерьвность на $\widetilde{S}$ функционала $J_{5}$ гарантирована при дополнительном условии $\eta \in C^{1}(\mathbb{R})$.

Функционал $J_{4}$ коэрцитивен на $\widetilde{S}$. Действительно, пусть $u_{k} \in \widetilde{S},\left\|u_{k}\right\|_{1} \rightarrow \infty, k \rightarrow \infty$. Выберем элемент $u_{y_{0}} \in H$, удовлетворяющий условию $u_{y_{0}}=y_{0}$ на $\Gamma_{0}$, и обозначим $\tilde{u}_{k}=u_{k}-u_{y_{0}}$. Очевидно, что $\left\|\tilde{u}_{k}\right\|_{1} \rightarrow \infty$. Тогда из неравенства Корна (см., например, [7]) вытекает, что $J_{4}\left(\tilde{u}_{k}\right) \rightarrow \infty$ при $k \rightarrow \infty$; следовательно, и $J_{4}\left(u_{k}\right) \rightarrow \infty$.

Если дополнительно предположить, что

$$
U_{a d} \subset\left\{g \in U: g_{\tau}=g-(g \cdot n) n=0 \quad \text { на } \Gamma_{1}\right\},
$$

то хорошо известно (см., например, [10]), что функционал $J_{2}$ коэрцитивен на $\widetilde{S}$.

Таким образом, имеет место

СлЕДСТВИЕ 3.1. Пусть выполняются условия (a), (b), $f \in L^{p}(\Omega), 1<p, \eta \in$ $C^{0}(\mathbb{R})$ и имеет место одно из следующих предположсений:

1) $J=J_{i}, i \in\{1,3\}, U_{a d}$ - ограниченное множество;

2) $J=J_{5}, \eta \in C^{1}(\mathbb{R}), U_{a d}$ - ограниченное мнохество;

3) $J=J_{4}$;

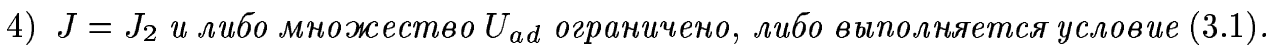

Тогда существует решение экстремальной задачи 1. 
3.3. Вывод системы оптимальности. Введем в рассмотрение операторы $\widetilde{C}^{T}$ : $H \times H \times H \rightarrow H^{*}, D: L^{\infty}(\Omega) \times H \times H \times H \rightarrow H^{*}, \widetilde{D}: H \times H \times H \times H \rightarrow H^{*}$, $F^{\prime}: H \times H \rightarrow H^{*}$, действующие по формулам

$$
\begin{gathered}
\left\langle F^{\prime}(u, w), y\right\rangle=\int_{\Omega} \eta^{\prime}(N u) w \cdot f N v d \Omega, \quad \widetilde{C}^{T}(u, w, v)=C^{T}(N(\eta(u)), w, v), \\
\langle D(\rho, u, w, v), y\rangle=\int_{\Omega} \rho(u \nabla) w \cdot v \cdot N y d \Omega, \\
\widetilde{D}(u, w, v, \phi)=D\left(\eta^{\prime}(N u), w, v, \phi\right) \quad \forall u, w, v, \phi \in H, \quad y \in H, \quad \rho \in L^{\infty}(\Omega) .
\end{gathered}
$$

Используя лемму 2.1 , неравенство Гёльдера и непрерывность вложений $(2.10)$, нетрудно показать, что $D(\tau, u, w, v), F^{\prime}(u, w) \in H^{*}$ для любых $\tau \in L^{\infty}(\Omega), u, w, v \in H$.

Лемма 3.1. Пусть $f \in \boldsymbol{L}^{p}(\Omega), 1<p, \eta \in C^{1}(\mathbb{R})$. Тогда операторы $\widetilde{D} u F^{\prime}$ усиленно непрерывные.

Доказательство проводится по такой же схеме, как и в лемме 2.2 .

Далее считаем, что

(с) функционал $J$ непрерывно диффференцируем по $\Phi$ реше.

Нетрудно проверить, что условие (с) выполняется для функционалов $J_{1}, J_{2}, J_{4}$, причем

$$
\begin{aligned}
& \left\langle J_{1}^{\prime}(u), v\right\rangle=\int_{\Omega}\left|u-u_{d}\right|^{s-2}\left(u-u_{d}\right) \cdot v d \Omega, \\
& \left\langle J_{2}^{\prime}(u), v\right\rangle=\int_{\Omega} \operatorname{rot} u \cdot \operatorname{rot} v d \Omega \quad \forall u, v \in H, \\
& \left\langle J_{4}^{\prime}(u), v\right\rangle=\mu \int_{\Omega}\left(\nabla u+(\nabla u)^{T}\right)\left(\nabla v+(\nabla v)^{T}\right) d \Omega .
\end{aligned}
$$

Если $\eta \in C^{1}(\mathbb{R})\left(\eta \in C^{2}(\mathbb{R})\right)$, то условие $\left(\right.$ с) вьполняется и для $J=J_{3}\left(J=J_{5}\right)$, причем

$$
\begin{aligned}
& \left\langle J_{3}^{\prime}(u), v\right\rangle=\int_{\Omega}\left|\eta(N u)-\rho_{d}\right|^{s-2}\left(\eta(N u)-\rho_{d}\right) \eta^{\prime}(N u) N v d \Omega \\
& \left\langle J_{5}^{\prime}(u), v\right\rangle=\int_{\Omega^{\prime}}\left|\eta^{\prime}(N u) u\right|^{s-2} \eta^{\prime}(N u) u \cdot\left(\eta^{\prime \prime}(N u) u N v+\eta^{\prime}(N u) v\right) d \Omega .
\end{aligned}
$$

ТЕОрема 3.2. Пусть выполняются условия (а)-(c), $f \in \boldsymbol{L}^{p}(\Omega), p>1, \eta \in C^{1}(\mathbb{R})$. Тогда для любого решения задачи $1\left(u_{*}, g_{*}\right)$ существуют не равные одновременно нулю әлементы $\left(w, \chi, \lambda_{0}\right) \in H_{0} \times U^{*} \times \mathbb{R}^{+}$такие, что

$$
\begin{gathered}
\mu\langle A w, v\rangle+\left\langle C^{T}\left(\eta\left(N u_{*}\right), u_{*}, w\right), v\right\rangle+\left\langle D\left(\eta^{\prime}\left(N u_{*}\right), u_{*}, u_{*}, w\right), v\right\rangle \\
+\left\langle\chi,\left.\gamma\right|_{\Gamma} v\right\rangle_{U^{*} \times U}+\lambda_{0}\left\langle J^{\prime}\left(u_{*}\right), v\right\rangle=\left\langle F^{\prime}\left(u_{*}, w\right), v\right\rangle \quad \forall v \in H, \\
\left\langle\chi, g_{*}-g\right\rangle_{U^{*} \times U}+\lambda \lambda_{0}\left(g-g_{*}, g_{*}-y_{d}\right)_{\Gamma_{1}} \geqslant 0 \quad \forall g \in U_{a d} .
\end{gathered}
$$


ДокАЗАтЕЛЬСтво. Пусть $\left(u_{*}, g_{*}\right)$ - решение задачи 1. Рассмотрим $\varepsilon$-задачу (метод адаптированного штрафа)

$$
J_{\varepsilon}(u, g) \rightarrow \inf , \quad u \in H, \quad g \in U_{a d}
$$

где

$$
\begin{aligned}
J_{\varepsilon}(u, g)= & J_{\lambda}(u, g)+\frac{1}{2}\left\|u-u_{*}\right\|_{1}^{2}+\frac{1}{2}\left\|g-g_{*}\right\|_{1 / 2, \Gamma_{1}}^{2} \\
& +\frac{1}{2 \varepsilon}\|\mu A u+\widetilde{B}(u, u, u)-F(u)\|_{H_{0}^{*}}^{2}+\frac{1}{2 \varepsilon}\|u-g\|_{1 / 2, \Gamma}^{2}, \quad \varepsilon>0 .
\end{aligned}
$$

Для простоты доказательства считаем, что $\left\|f_{1}\right\|_{H_{0}^{*}}=\left\|\nabla\left(A^{-1} f_{1}\right)\right\|_{\Omega}$ для любых $f_{1}$, $f_{2} \in H_{0}^{*}$. Используя лемму 2.2 , нетрудно показать, что существует решение $\left(u_{\varepsilon}, g_{\varepsilon}\right) \in$ $H \times U_{a d}$ задачи (3.4), причем $u_{\varepsilon} \rightarrow u_{*}$ сильно в $H, g_{\varepsilon} \rightarrow g_{*}$ сильно в $U$ при $\varepsilon \rightarrow+0$.

Запишем необходимые условия оптимальности первого порядка для решения $\left(u_{\varepsilon}, g_{\varepsilon}\right)$ задачи (3.4). Пусть $\theta \in[0,1]$,

$$
f_{1}(\theta)=J_{\varepsilon}\left(u_{\varepsilon}+\theta v, g_{\varepsilon}\right), \quad f_{2}(\theta)=J_{\varepsilon}\left(u_{\varepsilon}, g_{\varepsilon}+\theta\left(g-g_{\varepsilon}\right)\right), \quad v \in H, \quad g \in U_{a d} .
$$

Обозначим

$$
\begin{aligned}
& w_{\varepsilon}=\frac{1}{\varepsilon} A^{-1}\left(\mu A\left(u_{\varepsilon}\right)+\widetilde{B}\left(u_{\varepsilon}, u_{\varepsilon}, u_{\varepsilon}\right)-F\left(u_{\varepsilon}\right)\right) \in H_{0}, \\
& \chi_{\varepsilon}=\frac{1}{\varepsilon}\left(\left.\gamma\right|_{\Gamma}\left(u_{\varepsilon}-g\right)\right) \in L^{2}(\Gamma) \subset H^{-1 / 2}(\Gamma) \subset U^{*}
\end{aligned}
$$

Тогда из условий $f_{1}^{\prime}(0)=0, f_{2}^{\prime}(0) \geqslant 0$ вытекают соотношения

$$
\begin{aligned}
& \mu\left\langle A w_{\varepsilon}, v\right\rangle+\left\langle\widetilde{D}\left(u_{\varepsilon}, u_{\varepsilon}, u_{\varepsilon}, w_{\varepsilon}\right), v\right\rangle+\left\langle\chi_{\varepsilon},\left.\gamma\right|_{\Gamma} v\right\rangle+\left\langle J^{\prime}\left(u_{\varepsilon}\right), v\right\rangle \\
& \quad+\left(u_{\varepsilon}-u_{*}, v\right)_{1}+\left\langle\widetilde{C}^{T}\left(u_{\varepsilon}, u_{\varepsilon}, w_{\varepsilon}\right), v\right\rangle=\left\langle F^{\prime}\left(u_{\varepsilon}, w_{\varepsilon}\right), v\right\rangle \quad \forall v \in H, \\
& \left\langle\chi_{\varepsilon}, g_{\varepsilon}-g\right\rangle_{H^{-1 / 2}(\Gamma) \times H^{1 / 2}(\Gamma)}+\lambda\left(g-g_{\varepsilon}, g_{\varepsilon}-y_{d}\right)_{\Gamma_{1}} \\
& \quad+\left(g_{\varepsilon}-g_{*}, g-g_{\varepsilon}\right)_{1 / 2, \Gamma_{1}} \geqslant 0 \quad \forall g \in U_{a d} .
\end{aligned}
$$

Из уравнения (3.5) нетрудно получить, что $\left\|\chi_{\varepsilon}\right\|_{-1 / 2, \Gamma} \leqslant C_{1}\left\|w_{\varepsilon}\right\|_{1}$. Если $\left\|w_{\varepsilon}\right\|_{1} \leqslant C$, где постоянная $C$ не зависит от $\varepsilon$, то, переходя к пределу в $(3.5),(3.6)$, используя леммы 2.2 и 3.1 , получаем (3.2), (3.3), в которых следует положить $\lambda_{0}=1$. Пусть $m_{\varepsilon}=$ $\left\|w_{\varepsilon}\right\|_{1} \rightarrow \infty$. Положим $\widetilde{w}_{\varepsilon}=w_{\varepsilon} / m_{\varepsilon}$. Найдется подпоследовательность $\widetilde{w}_{\varepsilon_{n}} \rightarrow w$ слабо в $\boldsymbol{H}^{1}(\Omega)$. Если $w \neq 0$, то, разделив $(3.5),(3.6)$ на $m_{\varepsilon_{n}}$ и переходя к пределу при $\varepsilon_{n} \rightarrow+0$, получаем (3.2), (3.3), в которых $\lambda_{0}=0, w \neq 0$. Пусть $w=0$. Разделив (3.5), записанное для $\widetilde{w}_{\varepsilon_{n}}, u_{\varepsilon_{n}}$, на $m_{\varepsilon_{n}}$, выбрав $v=\widetilde{w}_{\varepsilon_{n}} \in H_{0}$ и использовав леммы $2.2,3.1$, приходим к соотношению

$$
\begin{gathered}
0<\mu \gamma \leqslant \mu\left\langle A \widetilde{w}_{\varepsilon_{n}}, \widetilde{w}_{\varepsilon_{n}}\right\rangle=-\left\langle\widetilde{C}\left(u_{\varepsilon_{n}}, \widetilde{w}_{\varepsilon_{n}}, u_{\varepsilon_{n}}\right), \widetilde{w}_{\varepsilon_{n}}\right\rangle-\left\langle\widetilde{D}\left(u_{\varepsilon_{n}}, u_{\varepsilon_{n}}, u_{\varepsilon_{n}}, \widetilde{w}_{\varepsilon_{n}}\right), \widetilde{w}_{\varepsilon_{n}}\right\rangle \\
-\frac{1}{m_{\varepsilon_{n}}}\left\langle J^{\prime}\left(u_{\varepsilon_{n}}\right), \widetilde{w}_{\varepsilon_{n}}\right\rangle-\frac{1}{m_{\varepsilon_{n}}}\left(u_{\varepsilon_{n}}-u_{*}, \widetilde{w}_{\varepsilon_{n}}\right)_{1}+\left\langle F^{\prime}\left(u_{\varepsilon_{n}}, \widetilde{w}_{\varepsilon_{n}}\right), \widetilde{w}_{\varepsilon_{n}}\right\rangle \rightarrow 0 .
\end{gathered}
$$

Противоречие. Следовательно, $w \neq 0$. Теорема доказана. 
СлЕДСТВИЕ 3.2. Пусть выполняются условия теоремы 3.2. Тогда существует постоянная $c_{1}=c_{1}(\Omega, p)$ такая, что если $u_{*}, g_{*}-$ решение задачи 1 , причем

$$
\mu>c_{1}\|\eta\|_{C^{1}}\left(\left\|u_{*}\right\|_{1}+\|f\|_{0, p}\left\|u_{*}\right\|_{1}+\left\|u_{*}\right\|_{1}^{2}\right)
$$

то существуют единственные $w \in H_{0}, \chi \in U^{*}$, удовлетворяющие уравнениям (3.2), (3.3), в которых следует положить $\lambda_{0}=1$.

ДокАЗАТЕЛЬСтво. Предположим, что $\lambda_{0}=0$. Выбирая в (3.2) $v=w$ и пользуясь соотношениями $(1.6),(2.9)$, нетрудно получить априорную оценку

$$
\mu\|w\|_{1}^{2} \leqslant c_{1}\|\eta\|_{C^{1}}\left(\left\|u_{*}\right\|_{1}^{2}+\|f\|_{0, p}\left\|u_{*}\right\|_{1}+\left\|u_{*}\right\|_{1}\right)\|w\|_{1}^{2} .
$$

Таким образом, если условие (3.7) выполнено, то $w=0$. В этом случае уравнение (3.2) принимает вид $\left\langle\chi,\left.\gamma\right|_{\Gamma} v\right\rangle=0$ для любого $v \in H$. Следовательно, и $\chi=0$, что противоречит теореме 3.1 .

ЗАмЕчАниЕ 1. Если в дополнение к условиям следствия $3.2 U_{a d}$ - ограниченноемножество (либо $J=J_{4}$ ), то из неравенств (2.11)-(2.15) вытекает, что найдутся постоянные $R, R_{1}$, зависящие только от исходных данных, такие, что

$$
\left\|g_{*}\right\|_{1 / 2, \Gamma} \leqslant R, \quad\left\|u_{*}\right\|_{1} \leqslant R_{1}
$$

причем $R_{1}=R_{1}(\nu) \rightarrow R_{1}^{\prime}<\infty$ при $\nu \rightarrow+\infty$. Следовательно, (3.7) вьполняется, если параметр $\nu=\mu /\|\eta\|_{C^{1}}$ достаточно большой.

Введем в рассмотрение оператор проектирования

$$
P_{R}: L^{2}(\Gamma) \rightarrow U_{a d}^{R}=U_{a d} \cap\left\{g \in H^{1 / 2}(\Gamma):\|g\|_{1 / 2, \Gamma} \leqslant R\right\} .
$$

Для того чтобы показать, что оператор $P_{R}$ определен на всем $L^{2}(\Gamma)$, достаточно установить, что выпуклое множество $U_{a d}^{R}$ замкнуто по норме $L^{2}(\Gamma)$. Действительно, пусть $g_{m} \in U_{a d}^{R}, g_{m} \rightarrow g$ в $L^{2}(\Gamma)$. Извлечем подпоследовательность $g_{m_{k}} \rightarrow \tilde{g} \in U_{a d}^{R}$ слабо в $H^{1 / 2}(\Gamma)$; вложение $H^{1 / 2}(\Gamma) \subset L^{2}(\Gamma)$ компактно; следовательно, $g_{m_{k}} \rightarrow \tilde{g}$ сильно в $L^{2}(\Gamma)$. Таким образом, $\tilde{g}=g \in U_{a d}^{R}$.

Если $\lambda \lambda_{0}>0$, то из соотношения (3.3) вытекает

$$
g_{*}=P_{R}\left(y_{d}-\frac{\chi}{\lambda \lambda_{0}}\right) .
$$

ЗАмечАниЕ 2. Соотношения (3.2), (3.3) (в которых полагаем $\lambda_{0}=1$ ) вместе с уравнениями

$$
\mu A u_{*}+\widetilde{B}\left(u_{*}, u_{*}, u *\right)=F\left(u_{*}\right),\left.\quad \gamma\right|_{\Gamma} u_{*}=g_{*}
$$

образуют по терминологии [6] сингулярную систему оптимальности.

ЗАмЕчАниЕ 3. Как уже отмечалось, в работе [7] исследована задача оптимизации функционала $J_{4}$ для уравнений Навье-Стокса однородной жидкости. В случае $\rho_{0}=$ const (т.е. жидкость однородная), $J=J_{4}$ основные результаты настоящей работы совпадают с полученными в [7]. 


\section{СПИСОК ЦИТИРОВАННОЙ ЛИТЕРАТУРЫ}

[1] Фролов Н. Н. О разрешимости краевой задачи движения неоднородной жидкости // Матем. заметки. 1993. Т. 53. №6. С. 130-140.

[2] Фролов Н.Н. Краевая задача, описьвающая движение неоднородной жидкости // Сиб. матем. ж. 1996. Т. 37. № 2. С. 433-451.

[3] Чеботарев А. Ю. Стационарные вариационные неравенства в модели неоднородной жидкости // Сиб. матем. ж. 1997. Т. 38. № 5. С. 1185-1193.

[4] Фурсиков А. В. Свойства решений некоторых экстремальных задач, связанных с системой Навье-Стокса // Матем. сб. 1982. Т. 118(180). №3(7). С. 323-349.

[5] Фурсиков А. В. Задачи управления и теоремы, касающиеся однозначной разрешимости смешанной краевой задачи для трехмерных уравнений Навье-Стокса // Матем. сб. 1981. T. 115. № 2. С. 281-306.

[6] Лионс ЖК.-Л. Управление сингулярными распределенньми системами.. М.: Наука, 1987.

[7] Gunzburger M. D., Hou L., Svobodny T. P. Boundary velocity control of incompressible flow with application to viscous drag reduction // SIAM J. Contr. Optim. 1992. V. 30. №1. P. 167-182.

[8] Темам Р. Уравнения Навье-Стокса. Теория и численньй анализ. М.: Мир, 1981.

[9] Треногин В. А. Функциональный анализ. М.: Наука, 1980.

[10] Conca C., Murat F., Pironneau O. The Stokes and Navier-Stokes equation with boundary conditions involving the pressure // Japan J. Math. 1994. V. 20. № 2. P. 279-318.

ИПМ ДВО РАН, г. Владивосток

Поступило

E-mail : cheb@ipm.marine.ru

05.09.1998

Исправленный вариант

29.08.2000 